\title{
The long-term effects of prolonged intensive care stay post-cardiac
}

\section{surgery}

\author{
Ursula Mackie-Savage $^{1}$ and Judith Lathlean ${ }^{2}$ \\ ${ }^{1}$ University College London Hospitals NHS Foundation Trust \\ ${ }^{2}$ University of Southampton
}

July 21, 2020

\begin{abstract}
Objectives: Short-term outcomes for those with a prolonged length of stay (LOS) in intensive care (ITU) following cardiac surgery are poor, with higher rates of in-hospital mortality and morbidity. Consequently, discharge from hospital has been considered the key measure of success. However, there has been a shift towards long-term outcomes, functional recovery and quality of life (QoL) as measures of surgical quality. The aim of this review is to compare and critique the findings of multiple studies in order to determine the long-term effects of prolonged ITU stay post-cardiac surgery. Methods: A computerised literature search of CINAHL, EMBASE and Google Scholar databases, based on keywords 'long-term effects'; 'prolonged ITU stay'; 'cardiac surgery', with rigorous CASP critique, was undertaken. Results: The search yielded 12 papers meeting the inclusion criteria, with eight retrospective and four prospective studies. Eight of these 12 papers identified inferior long-term survival or higher mortality rates for those who had prolonged LOS in ITU in comparison to 'normal' LOS or a control. The greatest burden of mortality was six months to one year post-discharge. Three papers found that quality of life was adversely affected or worse for those who had experienced prolonged LOS in ITU. Conclusions: Further research is required to provide better quality evidence into QoL, following prolonged stay in ICU post cardiac surgery. The evidence reviewed suggests that the risk of mortality in this demographic of patients is higher, especially within the first year and, therefore, more frequent medical surveillance of these patients is recommended.
\end{abstract}

\section{Introduction}

The burden of cardiovascular disease within a progressively ageing population has resulted in a shift in the demographic of cardiac surgery patients to include those that are older, increasingly frail and presenting with multiple co-morbidities ${ }^{(1),(2)}$. Despite a higher risk profile, mortality post-cardiac surgery remains low, due to continued advances in peri-operative care ${ }^{(3)}$. However, the complex nature of these patients means they are likely to encounter a longer and more complicated post-operative course, often involving a prolonged length of stay (LOS) in intensive care (ITU). Prolonged ITU stay has been reported as occurring in 4-11\% of cardiac patients (4) with other sources citing it to be as high as $36 \%{ }^{(5)}$. This poses both clinical and ethical issues as a very small proportion of patients are consuming an extremely high level of both human and financial resources. Care of the critically ill requires a high level of expenditure of time, money and resources; this includes specialist staff, one to one nursing care and sophisticated equipment and treatments (6). Critical care units across the UK are running at, or near full, capacity whilst also struggling with staffing shortages ${ }^{(7)}$. Lack of critical care bed availability often leads to cancellation of procedures, extension of waiting lists and compromised patient safety, thereby reducing operational performance across all areas of cardiac surgery and directly affecting patient care. Williams et al . ${ }^{(8)}$ identified a disproportionate usage of ITU beds in their study and concluded that the poor outcomes that have been reported after prolonged ITU stays may indicate it is neither beneficial to the patient nor cost effective. This is echoed by Gaudino et al. ${ }^{(9)}$ who commented that although life-saving treatment should not be withheld, resources should be 
allocated wisely to consider those waiting for treatment.

It is widely accepted that short-term outcomes for those with a prolonged LOS in ITU are poor, with higher rates of in-hospital mortality $(10 \%$ vs $0.6 \%)$ and morbidity ${ }^{(10),(11)}$. Additionally, both physical and cognitive impairments have been reported in those who have survived admission to an ICU, symptoms of which can persist for years following discharge, with more recent classification under the term 'post-intensive care syndrome' or PICS $^{(12)}$. Post-operative delirium in intensive care is a common occurrence in cardiac surgery patients $\left(26-52 \%^{(13)}\right)$ and there is evidence to suggest that those who experience delirium are at higher risk of long-term cognitive dysfunction ${ }^{(14)}$. This is compounded by the growing number of elderly patients undergoing surgery, with $37 \%$ of critically unwell adults over the age of 65 having pre-existing cognitive impairments such as dementia and depression ${ }^{(14)}$. PICS also encompasses physical impairments; muscle weakness as a result of critical care admission occurs in $40 \%$ of adult patients and in a small number of cases persists beyond discharge, resulting in poor functional ability and reduced quality of life. The mental health repercussions of ITU admission are perhaps the most marked, with $30 \%$ of patients experiencing depression, $70 \%$ anxiety and up to $50 \%$ demonstrating symptoms of post-traumatic stress disorder ${ }^{(14)}$.

Poorer outcomes for those with prolonged intensive care stay, increasing demand for critical care and a lack of resources has resulted in the development of enhanced recovery programmes. Enhanced recovery after surgery (ERAS) or 'fast track' programmes are a multidisciplinary approach that covers the entire patient journey (pre, intra and post-operatively) and have been designed to limit psychological and physiological stress in surgical patients in order to promote faster recovery ${ }^{(15)}$. Techniques include thorough preparation for surgery through patient assessment and education, minimally invasive surgical techniques, optimal fluid management and pain control and the early promotion of oral nutrition and mobilisation post-operatively ${ }^{(16)}$. ERAS was first implemented in colorectal surgery but its usage has spread to other specialities including cardiac surgery. The implementation of enhanced recovery pathways in cardiac surgery has been found to reduce not only LOS in hospital but also ITU LOS, post-operative complications and cost ${ }^{(17)}$. Coleman et al. (18) reported that although patient demographics, lifestyle and disease severity were similar between the ERAS and the control groups, the ERAS patients had better understanding of coronary artery disease, shorter fasting and water deprivation times, increased engagement with physiotherapy and improved physical performance post-operatively. Williams et al. ${ }^{(19)}$ saw a reduction in post-operative LOS by one day in the ERAS group and a reduction in intensive care unit LOS from 43 to 28 hours. Evidence demonstrating the positive effects of ERAS programmes within cardiac surgery, however, remains limited in comparison to other surgical specialties, and, as a consequence, ERAS is not yet widely implemented.

As a result of worse short-term clinical outcomes for patients with prolonged ITU stay, discharge from hospital was, until recently, considered the key measure of success. However, there has been a shift towards long-term outcomes, functional recovery and quality of life (QoL) as measures of surgical quality. There is now a body of primary research into long-term post-operative outcomes for cardiac patients with a focus on prolonged ICU stay, but it lacks consolidation. It is the aim of this review, therefore, to compare and critique the findings of multiple studies and provide an overview of the best available research on this topic in order to determine the long-term effects of prolonged ITU stay, and to inform and influence clinical practice in this area. Better understanding of the outcomes of this demographic of patients will also promote informed decision making for those considering cardiac surgery and allow clinicians to make more accurate decisions regarding treatment options, resource allocation and medical priorities. However in order to do so, greater understanding of prognosis, long-term survival and QoL is required.

\section{Methods}

A computerised literature search of CINAHL, EMBASE and Google Scholar was conducted. Published articles for inclusion in the review were identified using the following keywords/ search terms: 'long-term effects' and 'prolonged intensive care stay' or 'prolonged ITU stay' or 'prolonged ICU stay' and 'cardiac surgery'.

\section{Inclusion and Exclusion}


The search was refined by exclusion of any articles not written in English and any studies of paediatric patients. A timeframe of 2006-2020 was set to ensure that the reviewed evidence is relevant to current practice. After removal of duplicates and exclusion of papers that are not relevant because the participants were not adults and had not had cardiac surgery, this resulted in 12 papers (see appendix) for inclusion in the review.

\section{Critical Appraisal Methods}

The usage of a good quality critical appraisal tool allows a structured approach to the assessment of the rigor of the studies being reviewed ${ }^{(20)}$. The choice of an appropriate critical appraisal tool is considered to be an integral aspect of conducting a systematic review and of evidence-based practice ${ }^{(21)}$. Additionally, it is important to identify that, due to the wide variation in critical appraisal tools that are available, the quality of the evaluation that results from a review when using different tools will also vary, even when applied to the same literature. Design-specific tools are tailored to highlight potential methodological issues or bias that is unique to the study design; therefore The Critical Appraisal Skills Programme (CASP) tools for cohort studies (qualitative and quantitative) were chosen. CASP is a reputable organisation whose tools are free and easy to access, making this review easily reproducible and minimising the potential for varied evaluations of these pieces of literature.

\section{Results}

\section{Long-term survival and mortality}

Eight of the 12 papers reviewed identified inferior long-term survival or higher mortality rates for those who had prolonged LOS in ITU in comparison to 'normal' LOS or a control. Mahesh et al. ${ }^{(10)}$ reported overall survival at one year for prolonged ICU stay to be $89.2 \%$ vs $97.8 \%$ for the control group of LOS of less than three days, and $81.2 \%$ vs $93.6 \%$ at three year follow-up. Heimrath et al. ${ }^{(22)}$ reported mortality rates of $14 \%$ in the prolonged LOS group vs $4 \%$ for the control $(\mathrm{p}=0.001)$. Elfstrom et al. ${ }^{(23)}$ stated the mortality risk of groups with LOS 3-7 days and 7-14 days to be 50\% higher than that of the 'normal' LOS. High mortality rates for those with prolonged ITU LOS were also reflected in findings by Gaudino et al. ${ }^{(9)}$ where 57 participants survived to discharge and $78.9 \%$ of them died during follow-up (over an average of six years), of which 29 deaths were attributed to cardiac causes. However, it is important to note that this particular study had a small sample size $(\mathrm{n}=57)$ with no control population for comparison, therefore limiting generalisability of their findings to the wider population. Hassanet al. ${ }^{(24)}$ and Hein et al. ${ }^{(5)}$ reported poorer long-term survival in those with prolonged ITU stay that was more marked in the first six months to one year post-discharge. Long-term survival was $70 \%$ in the prolonged group vs. $90 \%$ in the control $(\mathrm{p}=<0.001)^{(24)}$ with similar results produced by Hein et al. ${ }^{(5)}$ with $66 \%$ survival in the prolonged group vs. $91 \%$ in the control $(\mathrm{p}=0.001)$. There was a steep decline in survival in the first six months of follow-up, but the six-month to three-year period was comparable (with a $7 \%$ difference in decline for the prolonged group vs. $4 \%$ for the control) which led the authors to conclude that chances of survival are comparable if they overcome the first six months post-operatively ${ }^{(5)}$. Similar findings and conclusions were made by Hassan et al. ${ }^{(24)}$ who demonstrated inferior long-term survival amongst patients with prolonged ITU LOS, especially within the first year after discharge, stating that the greatest burden of mortality is within the first year post-operatively.

Conversely, Silberman et al. ${ }^{(25)}$ found that a significant number of patients went on to experience reasonable long-term survival. Of those with LOS ranging from 1-2 days and 1-3 weeks, $90 \%$ were alive at one year follow-up. Eighty per cent of those with a LOS of one week and $50 \%$ of those with a LOS of two weeks were alive at five years. This was the only study to identify a proportional relationship between ITU LOS and overall late survival. They also found that for those with an extremely prolonged LOS in ITU (more than 30 days), the highest rate of mortality is in the first year. Manjiet al. ${ }^{(26)}$ took a different approach to their analysis by looking at 'functional survival' which they defined as patients being alive and not institutionalised. They found that the control group achieved $94.9 \%$ functional survival at one year vs. $73.9 \%$ for prolonged ICU stay and $84.9 \%$ vs. $53.8 \%$ at five years, which was considered a successful outcome by the authors. However, although the majority were alive and non-institutionalised, it is not known what QoL was like for 
these patients or whether they were requiring care or support at home as no assessment of QoL of functional status was made.

\section{Quality of Life}

Four of the 12 studies included assessment of quality of life in their research. Gaudino et al. ${ }^{(9)}$, Lagercrantzet al. ${ }^{(27)}$ and Barrie et al. ${ }^{(3)}$ all found that quality of life was adversely affected or worse for those who had experienced prolonged LOS in ITU. From a sample of 12 patients who survived to follow-up, only four patients had a Karnofsky score $>80$, indicative of having regained full autonomy post-operatively. Two patients were never discharged from a rehabilitation centre and six patients, although discharged home, were highly dependent on support with their activities of daily living (ADLs). Across all time intervals over the 6-84 month follow-up period, most patients had a low Karnofsky score $(<50)$ and a small number had intermediate scores (50-80). QoL (as measured by Karnofsky scores) did not improve over time ${ }^{(9)}$. Lagercrantz et al. ${ }^{(27)}$ reported higher scores, with $65 \%$ achieving $>80$ indicating full autonomy. However, they also demonstrated lower scores in both physical and mental domains for the study group vs their control (39.7 vs $43.6(\mathrm{p}=0.03)$ and 44.1 vs $50.8(\mathrm{p}=0.001)$ ). Barrie et al.$^{(3)}$ assessed QoL at $3-6$ months and one year time intervals. At 3-6 months the prolonged ICU stay group had greater weight loss (self-reported), greater fear of falling and deficits that were impacting their ability to drive. At one year, reported weight loss had improved but they were found to have deficits affecting their ability to carry out ADLs and limiting their walking distance. Those who underwent non-emergency cardiac surgery and had a subsequent prolonged LOS in ICU also reported higher 'decision regret' scores at 3-6 month and one year follow-up, compared to those who spent $<5$ days in ICU post-operatively. Initially the deficits affecting the ability to carry out ADLs were comparable; however by one year the QoL outcomes for the prolonged group were lower. On the other hand, Soppa et al. (28) found post-operative functional status in both Group A (ICU LOS 5-10 days) and Group B (ICU LOS > 10 days) was good, with mean Karnofsky scores of 87 vs 77.3; however no comparison was made against a control or 'normal' LOS group. The results reported by Barrie et al. ${ }^{(3)}$ should also be interpreted with some caution, due to a very small sample size $(n=35)$ and no baseline assessment of QoL pre-operatively and, therefore, it cannot be concluded with certainty that long-term QoL was impaired as a result of a prolonged ITU stay.

\section{Consideration of Ethics}

Five of the authors state that they received approval from their local ethics board for usage of patient data in their studies ${ }^{(3),(5),(22),(26),(27)}$ all of whom analysed the data retrospectively. The remaining seven authors do not mention having sought ethical approval. However, ethical approval is required for all studies involving identifiable patient data, so although not stated, it is assumed that this was received in all 12 studies in line with GDPR and Good Clinical Practice or they would have been unable to proceed to data collection and subsequent publication.

\section{Discussion}

Amongst the 12 papers identified, the definition of prolonged LOS varied widely from 2-14 days, therefore limiting comparability of the results. There were also variations in study design, firstly in the usage of a comparison or control group. The most common method of comparison was using a 'normal' LOS group versus a 'prolonged' LOS group; however a lack of standardised definition of 'prolonged' means that, although similar methodology was used, results may differ widely. Silbermanet al. ${ }^{(25)}$ considered normal LOS to be $<2$ days, therefore used ITU LOS of $<2$ days as the control vs the comparison groups of 2-14 days and $>14$ days, whereas Hein et al. ${ }^{(5)}$ classified 'prolonged' to be LOS exceeding 14 days and used the results as a comparison for LOS $<14$ days. One study used an age-sex matched group from the general population for comparison, and not patients who had undergone cardiac surgery ${ }^{27)}$. This too limits comparability between the groups as there may be multiple variables unaccounted for, which may have impacted long-term survival and QoL, and this also goes against a key premise of the cohort study, that both exposed and unexposed groups must be taken from the same source population ${ }^{(30)}$. Two papers made no comparison to a control ${ }^{(9)}$,

(26) so external factors cannot be ruled out when considering their results. Soppa et al. ${ }^{(28)}$ described having 
used an 'internal control' but it is not clear what this constitutes. Comparisons were made between 'Group A' with LOS of 5-10 days and 'Group B' of LOS $>10$ days but the authors do not clarify whether both groups are considered to have experienced prolonged stay. It would seem that they are to be classified as having had a prolonged LOS, as only $4.7 \%$ of the 2250 cohort were categorised into Group A or B, indicating that the remaining $95.3 \%$ either had an uneventful post-operative course and subsequent discharge from ITU or died post-operatively, although why comparison was not made with those of 'normal' ITU LOS instead is not addressed by the authors. Secondly, there was wide disparity in sample sizes between studies. Silberman et al. ${ }^{(25)}$ recruited a large cohort of 6385 patients in comparison to Soppa et al. ${ }^{(28)}$ with 108 patients and Barrie et al. ${ }^{(3)}$ with just 35 patients matched with 35 control participants. It seems that Silberman et al. ${ }^{(25)}$ achieved this through a wide window of data collection, retrospectively identifying all patients undergoing cardiac surgery between 1993-2011. On the other hand, although Soppa et al. ${ }^{(28)}$ identified 2250 cardiac surgery patients, only 108 participants met the inclusion criteria as only $4.7 \%$ experienced prolonged LOS in ICU. The advantages of a large sample size are that they can provide greater precision of estimation of treatment effects and are more likely to be representative of the sample, thus improving generalisability of the results. Sample size is also a key determining factor for the risk of generating false positive or false negative results ${ }^{(29)}$. However, it can be argued that, on the basis of statistics, there is no reason why a significant result from a cohort of 6385 should be trusted more than a result from a cohort of 108, given the level of significance is the same. Biau et al. ${ }^{(29)}$ also commented that small trials, if well designed, can still produce reliable estimation of treatment effect; however this is in reference to RCTs which are designed to mitigate the effects of external variables and bias through randomisation and blinding, which is not achievable in cohort studies.

Cohort studies are either prospective or retrospective in design; the most common approach amongst these pieces of literature was retrospective (employed in seven of the 12 studies). Retrospective cohort studies are less costly and less time consuming than prospective to conduct, but they are also more susceptible to bias $^{(30)}$. Firstly, with studies with lengthy follow-up periods of months to years, such as these, there is a risk of attrition bias that occurs from loss to follow-up through death, migration, late refusal to participate or losses that occur as a result of the exposure itself ${ }^{(30)}$. In this instance there may have been impairments postsurgery that could limit an individual's ability to participate in long-term follow-up, resulting in potential bias and consequent skewing of data. Most studies achieved $100 \%$ follow up of their participants, although this was easier to achieve for those which were retrospective in design. Those that looked at QoL requiring the completion of questionnaires were less successful at obtaining complete follow-up. Lagercrantz et al. (27) achieved $72 \%$ response rates and Barrie et al. ${ }^{(3)}$ reported a $17 \%$ loss of the prolonged group participants and a $23 \%$ loss of participation from the comparison group, which is higher than what is considered to be an acceptable level of loss (below 20\%) ${ }^{(30)}$. Secondly, when there is disparity between completeness of followup between the exposure group and control or comparison group, this can lead to selection bias ${ }^{(30)}$ which occurred in one study where a higher proportion of phone survey responses were received in the control $\operatorname{group}^{(3)}$.

QoL was assessed in four of the studies using Karnofsky's Performance Status scale (KPS), the SP-36 and a combination of the New York Heart Association classification, frailty assessments and the Hospital Anxiety and Depression Scale. Usage of different tools will undoubtedly yield different results; however at present there is no approved cardiac-specific QoL assessment tool. The KPS scale, which was used in two of the studies, has been criticised for lacking sensitivity at the lower end of the spectrum, resulting in inaccurate classification of functional status for patients with greater physical impairments ${ }^{(31)}$. It also does not include the assessment of mental health which means it does not address the psychological burden that can result from ICU stay, the effects of which have been shown to still be evident years following discharge ${ }^{(14)}$. The KPS scale was also designed for usage by oncology patients and, therefore, due to the lack of a cardiac specific tool, a generic assessment tool such as the SF-36 may have been more appropriate for this demographic of patients. However, although more widely applicable, generic tools do not include all of the dimensions relevant to a specific patient group, consequently reducing their sensitivity ${ }^{(32)}$ and increasing the possibility of missing factors which may be key to the patient's perception of their QoL. Additionally, no baseline 
assessment of pre-operative QoL was made in any of the four studies which means it is not possible to conclude that QoL was impaired as a result of their treatment or prolonged LOS in ITU as the impairments may have been pre-existing or as a result of a comorbidity. Impaired QoL pre-operatively has been shown to be a strong predictor of impaired QoL late after cardiac surgery ${ }^{(32)}$ and therefore may have affected the results. Manji et al. ${ }^{(26)}$ reported good functional survival at one and five years post-operatively, i.e more than $50 \%$ of participants were alive and non-institutionalised. However, although alive, it is not possible to ascertain whether they would have reported an acceptable QoL or whether they were highly dependent and requiring care. Analysis of long-term survival or mortality was found to be significant in all 12 studies; however those utilising Karnofsky as a measure of QoL either did not include the statistical analysis in the published $\operatorname{article}^{(3)}$ or the results were not statistically significant ${ }^{(27)}$. Lagercrantz et al. ${ }^{(27)}$ used the SF-36 in conjunction with Karnofsky as a QoL measure and the SF-36 results were found to be statistically significant. Length of follow-up was adequate for the assessment of long-term outcomes in all of the studies reviewed, ranging from six months to ten years. Although the way in which cardiac surgery is performed has not changed dramatically in the last decade, improvements to pre, intra and post-operative care continue to be made in a bid to improve outcomes, changes which may have influenced ICU practices and ultimate timing of patient discharge from ICU. In an effort to mitigate this bias, standard operating procedures and local policies were reported to have been followed ${ }^{(5)}$.

\section{Conclusions and Implications for Clinical Practice}

The findings demonstrate that long-term survival is worse for those who have experienced prolonged ICU LOS $(23)(9),(24)(5),(22)(27),(10)$. Notably, mortality is highest in the first six months to one year post-discharge, indicating that more comprehensive follow-up and surveillance is required in this time ${ }^{(5),(10),(24),(25)}$. Followup of cardiac surgery patients typically occurs $6-12$ weeks post-operatively ${ }^{(33)}$; however the timing of this is determined locally and there is currently no national guidance regarding this. NHS England advises that, upon discharge, patient care is transferred to their local district general hospital or GP for medical review and referral for cardiac rehabilitation services (if appropriate) ${ }^{(34)}$. Consequently, the onus falls on the GP and the patient themselves to identify and report any complications, deficits or long-term effects of prolonged ITU stay. The traditional approach of six week follow-up is also not evidence-based ${ }^{(35)}$ and those with a prolonged stay in ITU are not flagged as higher risk and therefore are not followed up more frequently nor comprehensively. The findings of this review suggest that greater surveillance of patients with prolonged LOS in ITU particularly in the first six months to one year is likely to be beneficial. Mahesh et al. (10) found prolonged ICU LOS to be an independent predictor of shorter long-term survival and Silberman et al. (25) identified a proportional relationship between ITU LOS and survival. Due to a lack of good quality and statistically significant findings, it is not possible to determine whether QoL is also adversely affected by prolonged ICU LOS. Further research into QoL outcomes for this demographic is undoubtedly required since, as the population continues to age, more patients will undergo cardiac surgery and survive to discharge, making it crucial to understand what the long-term outcomes are for those requiring prolonged ICU LOS. Improved understanding of post-operative QoL for cardiac surgery patients will enable clinicians to better support informed decision making and provide realistic post-operative expectations to patients and their families. The findings of this review also highlight the importance of efforts to reduce LOS in ITU, such as the implementation of ERAS programmes.

\section{Limitations}

There are limitations of this review that require acknowledgement. Firstly, widening the search period to allow for research pre 2006 would have resulted in more papers eligible for inclusion. However, due to resourcing and time restraints, review of more than 12 papers was not manageable and was beyond the scope of this review. Excluding papers on a language basis (only including those written in English) may have also impacted the findings of this review and high quality and relevant findings may have inadvertently been missed as a result of the inclusion/exclusion criteria. Lastly, as previously identified, the findings of the review are in part dependent on the choice of critical appraisal tool and the application of other critical appraisal tools to the same literature may have yielded different results. 
Summary Table
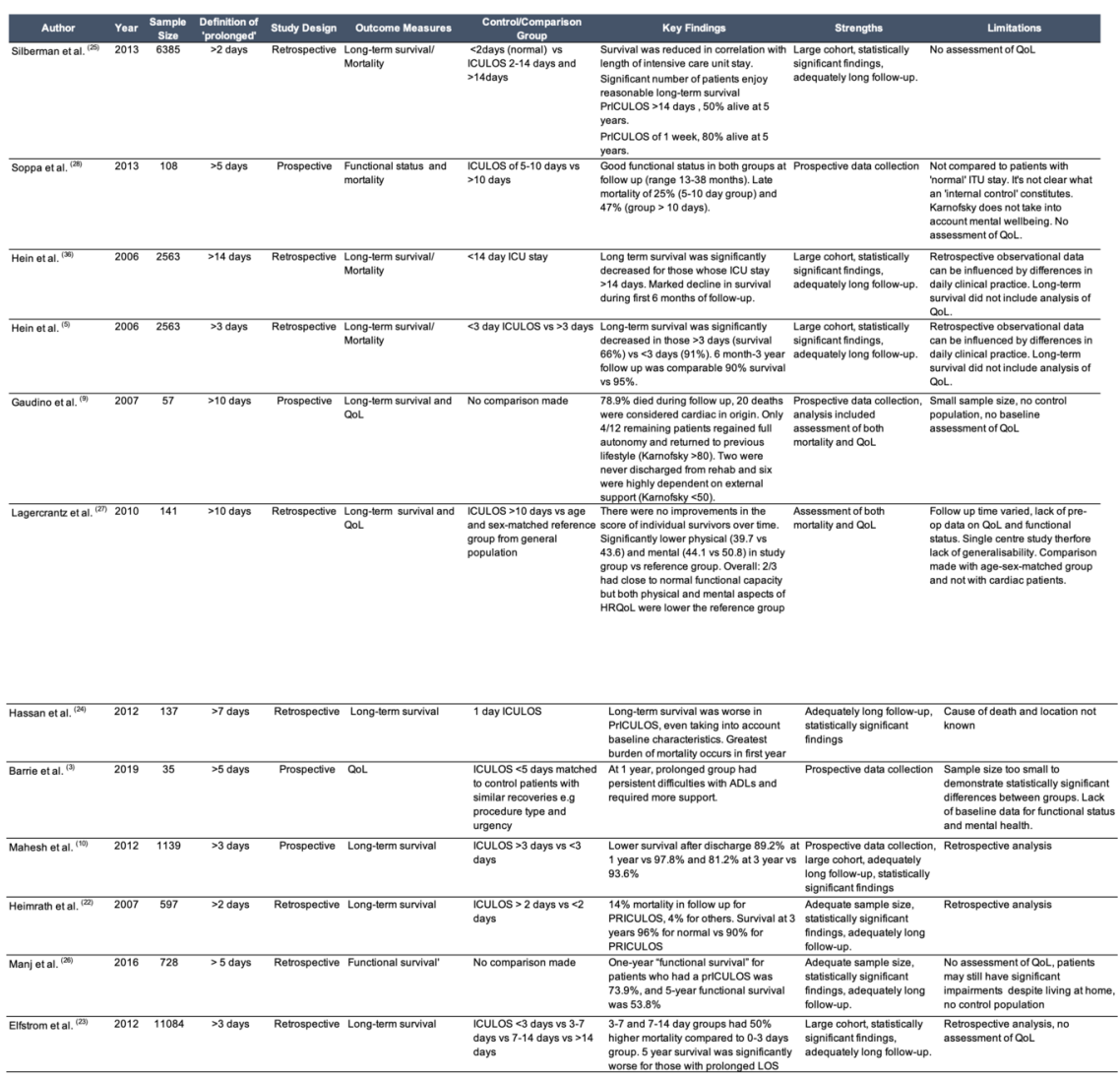

Author Contributions

Ursula Mackie-Savage was responsible for designing the research question, for conducting the literature search, for article review, subsequent analysis and for drafting of the paper. Judith Lathlean assisted throughout the process including the data analysis, drafting of the paper and critical revisions leading to its final publication.

\section{References}

Stephens R, Whitman G. Postoperative Critical Care of the Adult Cardiac Surgical Patient. Critical Care Medicine. 2015;43(9):1995-2014.

1. Kapadohos T, Angelopoulos E, Vasileiadis I, Nanas S, Kotanidou A, Karabinis A et al. Determinants of prolonged intensive care unit stay in patients after cardiac surgery: a prospective observational study. Journal of Thoracic Disease. 2017;9(1):70-79.

2. Barrie K, Cornick A, Debreuil S, Lee E, Hiebert B, Manji R et al. Patients With a Prolonged Intensive Care Unit Length of Stay Have Decreased Health-Related Quality of Life After Cardiac Surgery. Seminars in Thoracic and Cardiovascular Surgery. 2019;31(1):21-31.

3. Diab M, Bilkhu R, Soppa G, McGale N, Hirani S, Newman S et al. Quality of Life in Relation to Length of Intensive Care Unit Stay After Cardiac Surgery. Journal of Cardiothoracic and Vascular Anesthesia. 2017;31(3):1080-1090.

4. Hein O, Birnbaum J, Wernecke K, England M, Konertz W, Spies C. Prolonged Intensive Care Unit Stay in Cardiac Surgery: Risk Factors and Long-Term-Survival. The Annals of Thoracic Surgery. 2006; $81(3): 880-885$. 
5. Seidel J, Whiting P, Edbrooke D. The costs of intensive care. Continuing Education in Anaesthesia Critical Care \& Pain. 2006;6(4):160-163.

6. Faculty of Intensive Care Medicine. Critical Capacity: A short survey on critical care bed capacity [Internet]. The Faculty of Intensive Care Medicine. 2018 [cited 16 March 2020]. Available from: https://www.ficm.ac.uk/sites/default/files/ficm_critical_capacity_-_a_short_research_survey_on_critical_care_bed_capacity.pdf

7. Williams M, Wellner R, Hartnett E, Thornton B, Kavarana M, Mahapatra R et al. Long-term survival and quality of life in cardiac surgical patients with prolonged intensive care unit length of stay. The Annals of Thoracic Surgery. 2002;73(5):1472-1478.

8. Gaudino M, Girola F, Piscitelli M, Martinelli L, Anselmi A, Della Vella C et al. Long-term survival and quality of life of patients with prolonged postoperative intensive care unit stay: Unmasking an apparent success. The Journal of Thoracic and Cardiovascular Surgery. 2007;134(2):465-469.

9. Mahesh B, Choong C, Goldsmith K, Gerrard C, Nashef S, Vuylsteke A. Prolonged Stay in Intensive Care Unit Is a Powerful Predictor of Adverse Outcomes After Cardiac Operations. The Annals of Thoracic Surgery. 2012;94(1):109-116.

10. Schoffelen A, Hofhuis J, Rommes J, Spronk P. Consumption of ICU resources by long-stay patients does not change over time: 10-year observation in a teaching hospital in The Netherlands [Internet]. CyberLeninka. 2008 [cited 15 March 2020]. Available from: https://cyberleninka.org/article/n/192393

11. Colbenson G, Johnson A, Wilson M. Post-intensive care syndrome: impact, prevention, and management. Breathe. 2019;15(2):98-101.

12. Brown C. Delirium in the cardiac surgical ICU. Current Opinions in Anaesthesiology. 2014;27(2):177122.

13. Inoue S, Hatakeyama J, Kondo $\mathrm{Y}$, Hifumi T, Sakuramoto H, Kawasaki $\mathrm{T}$ et al. Post-intensive care syndrome: its pathophysiology, prevention, and future directions. Acute Medicine \& Surgery. 2019;6(3):233-246.

14. Ljungqvist O, Hubner M. Enhanced recovery after surgery - ERAS - principles, practice and feasibility in the elderly. Aging Clinical and Experimental Research. 2018;30(3):249-252.

15. Simpson J, Moonesinghe S, Grocott M, Kuper M, McMeeking A, Oliver C et al. Enhanced recovery from surgery in the UK: an audit of the enhanced recovery partnership programme 2009-2012. British Journal of Anaesthesiology. 2015;115(4):560-568.

16. Li M, Zhang J, Gan T, Qin G, Wang L, Zhu M et al. Enhanced recovery after surgery pathway for patients undergoing cardiac surgery: a randomized clinical trial. European Journal of Cardio-Thoracic Surgery. 2018;54(3):491-497.

17. Coleman S, Chen M, Patel S et al. Enhanced Recovery Pathways for Cardiac Surgery. Curr Pain Headache Rep. 2019;23(4)

18. Williams J, McConnell G, Allender J, Woltz P, Kane K, Smith P et al. One-year results from the first US-based enhanced recovery after cardiac surgery (ERAS Cardiac) program. The Journal of Thoracic and Cardiovascular Surgery. 2019;157(5):1881-1888.

19. Smith J, Noble H. Reviewing the literature: Table 1. Evidence Based Nursing. 2015;19(1):2-3.

20. Katrak P, Bialocerkowski A, Massy-Westropp N, Kumar V, Grimmer K. A systematic review of the content of critical appraisal tools. BMC Medical Research Methodology. 2004;4(1).

21. Heimrath $\mathrm{O}$, Buth K, Légaré J. Long-term outcomes in patients requiring stay of more than 48 hours in the intensive care unit following coronary bypass surgery. Journal of Critical Care. 2007;22(2):153-158.

22. Elfstrom K, Hatefi D, Kilgo P, Puskas J, Thourani V, Guyton R et al. What Happens After Discharge? An Analysis of Long-Term Survival in Cardiac Surgical Patients Requiring Prolonged Intensive Care. Journal of Cardiac Surgery. 2011;27(1):13-19.

23. Hassan A, Anderson C, Kypson A, Kindell L, Ferguson T, Chitwood W et al. Clinical Outcomes in Patients With Prolonged Intensive Care Unit Length of Stay After Cardiac Surgical Procedures. The Annals of Thoracic Surgery. 2012;93(2):565-569.

24. Silberman S, Bitran D, Fink D, Tauber R, Merin O. Very Prolonged Stay in the Intensive Care Unit After Cardiac Operations: Early Results and Late Survival. The Annals of Thoracic Surgery. 
2013;96(1):15-22.

25. Manji R, Arora R, Singal R, Hiebert B, Moon M, Freed D et al. Long-Term Outcome and Predictors of Noninstitutionalized Survival Subsequent to Prolonged Intensive Care Unit Stay After Cardiac Surgical Procedures. The Annals of Thoracic Surgery. 2016;101(1):56-63.

26. Lagercrantz E, Lindblom D, Sartipy U. Survival and Quality of Life in Cardiac Surgery Patients With Prolonged Intensive Care. The Annals of Thoracic Surgery. 2010;89(2):490-495.

27. Soppa G, Woodford C, Yates M, Shetty R, Moore M, Valencia O et al. Functional status and survival after prolonged intensive care unit stay following cardiac surgery+. Interactive CardioVascular and Thoracic Surgery. 2013;16(6):750-754.

28. Biau D, Kerneis S, Porcher R. Statistics in Brief: The Importance of Sample Size in the Planning and Interpretation of Medical Research. Clinical Orthopaedics and Related Research. 2008;466(9):22822288 .

29. Song J, Chung K. Observational Studies: Cohort and Case-Control Studies. Plastic and Reconstructive Surgery. 2010;126(6):2234-2242.

30. Kelly C, Shahrokni A. Moving beyond Karnofsky and ECOG Performance Status Assessments with New Technologies. Journal of Oncology. 2016;2016:1-13.

31. De Smedt D, Clays E, De Bacquer D. Measuring health-related quality of life in cardiac patients: Table 1. European Heart Journal - Quality of Care and Clinical Outcomes. 2016;2(3):149-150.

32. After Your Heart Operation [Internet]. Royal Brompton and Harefield NHS Trust. [cited 14 July 2020]. Available from: https://www.rbht.nhs.uk/sites/nhs/files/PILs/After\%20your\%20heart\%20operation\%20\%20Royal\%20Brompton\%20Hospital\%20-\%20January\%202018.pdf

33. Service Specifications: Cardiac Surgery- Adults [Internet]. NHS England. 2015 [cited 14 July 2020]. Available from: https://www.england.nhs.uk/commissioning/wpcontent/uploads/sites/12/2015/01/a10-spec-adlt-cardiac-surgry.pdf

34. Ngaage D, Gooseman M, Bulliment K, Jarvis M, Chaudhry M, Cale A et al. Is six weeks too long for the first outpatient review after cardiac surgery? FORCAST6. British Journal of Cardiology. $2019 ; 26(34)$

35. Hein O, Birnbaum J, Wernecke K, Konertz W, Spies C. Intensive Care Unit Stay of More than 14 Days after Cardiac Surgery is Associated with Non-cardiac Organ Failure. Journal of International Medical Research. 2006;34(6):695-703.

\section{Appendix}

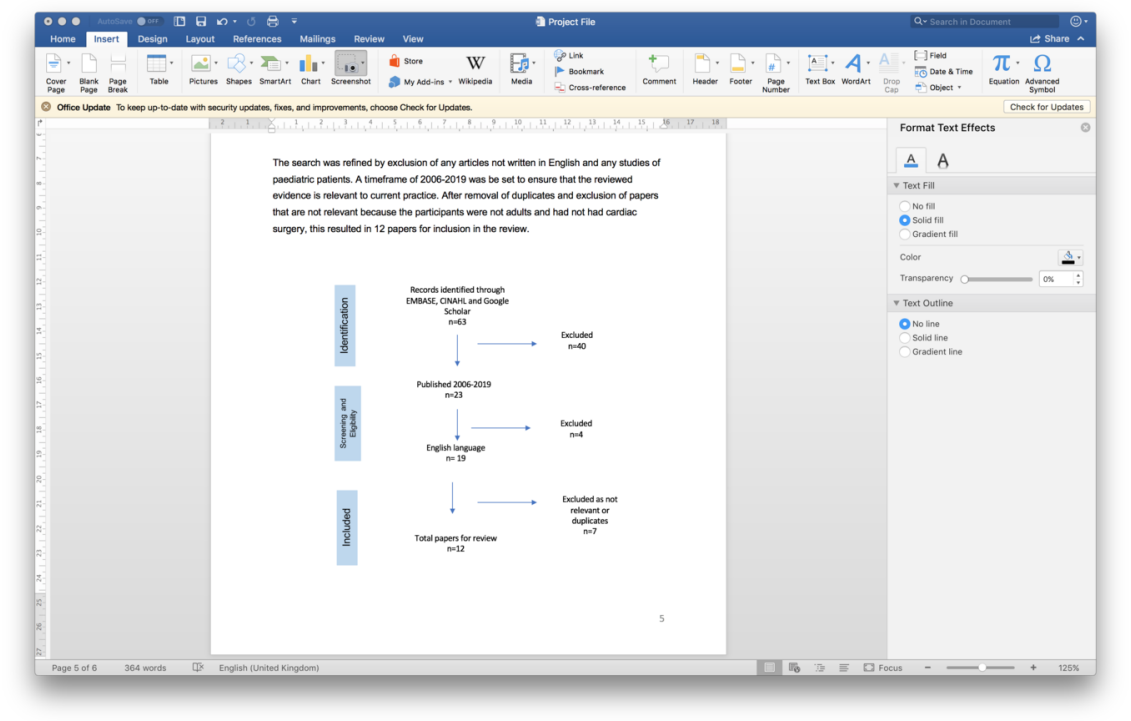


PRISMA Flow Diagram 